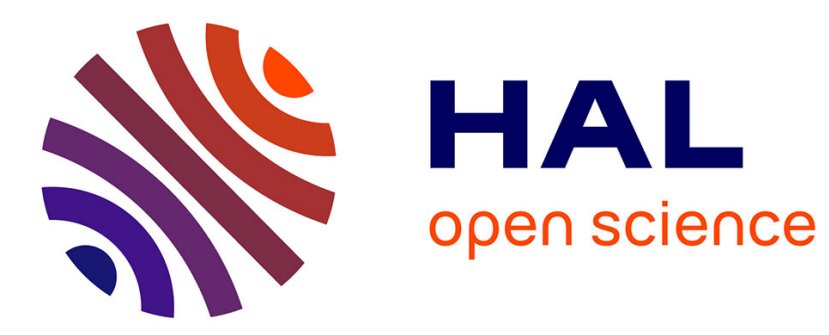

\title{
Resolving the crossing/kissing fiber ambiguity using Functionally Informed COMMIT
}

Matteo Frigo, Isa Costantini, Rachid Deriche, Samuel Deslauriers-Gauthier

\section{To cite this version:}

Matteo Frigo, Isa Costantini, Rachid Deriche, Samuel Deslauriers-Gauthier. Resolving the crossing/kissing fiber ambiguity using Functionally Informed COMMIT. Computational Diffusion MRI 2018, Sep 2018, Granada, Spain. 10.1007/978-3-030-05831-9 . hal-01864939

\section{HAL Id: hal-01864939 \\ https://hal.inria.fr/hal-01864939}

Submitted on 30 Aug 2018

HAL is a multi-disciplinary open access archive for the deposit and dissemination of scientific research documents, whether they are published or not. The documents may come from teaching and research institutions in France or abroad, or from public or private research centers.
L'archive ouverte pluridisciplinaire HAL, est destinée au dépôt et à la diffusion de documents scientifiques de niveau recherche, publiés ou non, émanant des établissements d'enseignement et de recherche français ou étrangers, des laboratoires publics ou privés. 


\title{
Resolving the crossing/kissing fiber ambiguity using Functionally Informed COMMIT
}

\author{
Matteo Frigo, Isa Costantini, Rachid Deriche, Samuel Deslauriers-Gauthier \\ ATHENA Project Team, Inria Sophia-Antipolis Mediterranée \\ Université Côte D'Azur, France
}

\begin{abstract}
The architecture of the white matter is endowed with kissing and crossing bundles configurations. When these white matter tracts are reconstructed using diffusion MRI tractography, this systematically induces the reconstruction of many fiber tracts that are not coherent with the structure of the brain. The question on how to discriminate between true positive connections and false positive connections is the one addressed in this work. State-of-the-art techniques provide a partial solution to this problem by considering anatomical priors in the false positives detection process. We propose a novel model that tackles the same issue but takes into account both structural and functional information by combining them in a convex optimization problem. We validate it on two toy phantoms that reproduce the kissing and the crossing bundles configurations, showing that through this approach we are able to correctly distinguish true positives and false positives.
\end{abstract}

Keywords: Tractography · False positives · Diffusion MRI - Resting state functional MRI.

\section{Introduction}

The increasing interest in connectomics shed light on the intrinsic limitations of tractography based connectomes $[19,13,6]$. Tractography itself is an ill-posed problem that aims at reconstructing the white matter fiber tracts from diffusion MRI (dMRI) data and is shown to generate a significant number of false positive connections between brain regions [13]. This is due to the fact that whenever two bundles of axons are organized in a crossing or kissing configuration, in-vivo tractography is not able to distinguish their actual trajectories from diffusion data. This implies the presence of many spurious entries in the structural connectome obtained from a tractogram. Moreover, specificity of brain networks has been demonstrated to be twice as important as sensitivity when estimating properties of the brain networks [19]. The re-establishment of an equilibrium between highly sensitive and highly specific networks is a challenge in brain connectomics and is the goal of this work. The task of extracting only true positive connections from a tractogram is also called tractography optimization or tractogram filtering $[8,9]$. Several tools to perform this task have been proposed in the last years, including SIFT2 [17], LiFE [15] and COMMIT [7]. While the 
first has the goal of matching a tractogram with the fiber densities estimated using the spherical deconvolution diffusion model, the two last are designed in such a way that they identify a subset of streamlines that explains the acquired diffusion MRI through a linear forward model. This model takes into account only the intra-cellular compartment in the case of LiFE and more complex tissue microstructure models in the case of COMMIT. Recent studies showed that anatomical priors can be taken into account while detecting false positives [8]. Daducci et al. [5] analyzed how the intrinsic bundle structure of both the brain and tractograms can be used to tackle the false positives issue, while Girard et al. [10] exploited microstructural information to solve the kissing bundles problem. Still, both these approaches showed some limitations. What we propose in this work is to overtake the purely structural-based approach and consider both structural and functional information in the formulation of the tractography optimization problem. We do this by showing that the injection of functional priors coming from resting-state functional MRI (rs-fMRI) gives an effective answer to the false positives issue. The functional information is exploited in the form of static functional connectivity and it plays the role of promoting the involvement of bundles that connect highly correlated cortical regions in the fitting of the dMRI signal. We encapsulate all of this within the COMMIT framework, which provides a flexible tool for defining a convex optimization problem that, for a given tractogram, simultaneously promotes sparsity among the bundles, takes into account the considered functional information and selects the streamlines that are sufficient to explain the diffusion MRI signal. We call this novel framework Functionally Informed COMMIT (FIC). This formulation is tested on TVB [16] and Phantomas [3] based synthetic phantom datasets that reproduce the two problematic configurations of crossing and kissing bundles, which are the source of most of the biases in connectivity estimation.

\section{Theory}

As the name suggests, tractography optimization implies the solution of a minimization problem. The functional to be minimized takes into account both the structural information coming from dMRI and the functional information given by rs-fMRI. The space in which we want to optimize it is the space of streamlines, hence we want to assign to each of them a coefficient $x_{i} \geq 0$. This coefficient quantifies the contribution of streamline $i$ to the definition of the dMRI signal, which means that if $x_{i}=0$ then the $i$-th streamline does not explain the diffusion signal and will be marked as a false positive. The fitting of the dMRI signal is tackled by means of the COMMIT framework. In particular, we use the provided linear forward model for diffusion MRI that predicts the signal generated by a tractogram through a given microstructure model. The one we are going to consider during this work is the Stick-Zeppelin-Ball model [14]. This leads to the definition of the optimization problem as

$$
x^{*}=\underset{x \in \mathbb{R}_{+}^{n}}{\operatorname{argmin}} \frac{1}{2}\|A x-y\|_{2}^{2}+\Omega(x)
$$


where $n$ is the number of streamlines, $y \in \mathbb{R}^{m}$ is the vectorized dMRI signal, $A \in \mathbb{R}^{m \times n}$ is the linear operator that projects streamline weights on the diffusion data according to the microstructure model and $\Omega: \mathbb{R}^{n} \rightarrow \mathbb{R}$ is a regularization term that for $\Omega(x)=0$ returns the classical formulation of COMMIT, which will be used as a first reference for our results.

Regularization term. Our objective is to promote sparsity in a structured way, namely we want to promote the preservation of the streamline bundles, instead of individual streamlines, that are sufficiently significant to explain the diffusion MRI signal. This leads to the definition of the following regularization term

$$
\Omega(x)=\lambda \sum_{g \in \mathcal{G}} w_{g}\left\|x_{g}\right\|_{2}
$$

where $\mathcal{G}$ is a collection of groups of indexes (i.e. streamline bundles) forming a partition of $[1, n], w_{g}$ is a positive weight associated to each group $g, x_{g}$ is the restriction of $x \in \mathbb{R}^{n}$ to the entries indexed by $g$ and $\lambda \geq 0$ is the regularization parameter. This $\Omega$ is a classical choice [1] in structured sparsity regularization and is also known as $\ell_{1,2}$ regularization. The groups can be both decoupled and overlapping, leading to different strategies in the solution of the optimization problem. See [11] for an exhaustive discussion on the mathematical details of this formulation and [8] for an application of this concept in tractography optimization. A common choice for the specific weight associated to each group is $N_{g}^{-1 / 2}$, where $N_{g}$ is the cardinality of group $g$. This normalization avoids preserving a group just because it involves a smaller number of variables. In the context of our problem this means that we try to avoid penalizing bigger bundles more than smaller bundles. This choice of $w_{g}$ is called group sparsity and will be used as a second reference to compare our results. Notice that group sparsity is purely based on a structural prior, hence it does not take into account the fact that a bundle could connect two regions that are more or less functionally correlated. We can quantify the functional correlation between cortical regions $i$ and $j$ by means of the functional correlation coefficient $c_{i, j}$. If bundle $g$ connects regions $i$ and $j$, we associate to it the coefficient $c_{g}=c_{i, j}$. How to compute this functional correlation coefficient is the question addressed in Section 3.1. Our objective is to favor more bundles that are associated to high functional correlation than the ones associated to low functional correlation. We designed a strategy for determining the weight $w_{g}$ that reads as follows:

$$
w_{g}=\frac{1}{\sqrt{N_{g}}}\left(\frac{1}{c_{g}^{2}} \exp \left(\frac{1-c_{g}^{2}}{2 s^{2}}\right)-1\right) \text {. }
$$

The function of $c_{g}$ defined in equation (3) enjoys the property of being equal to zero when $c_{g}=1$ and going asymptotically to infinity when $c_{g} \rightarrow 0^{+}$. The undefined parameter $s$ controls the speed with which $w_{g}$ goes to infinity. In particular it affects more the behavior in the case of low functional correlation 


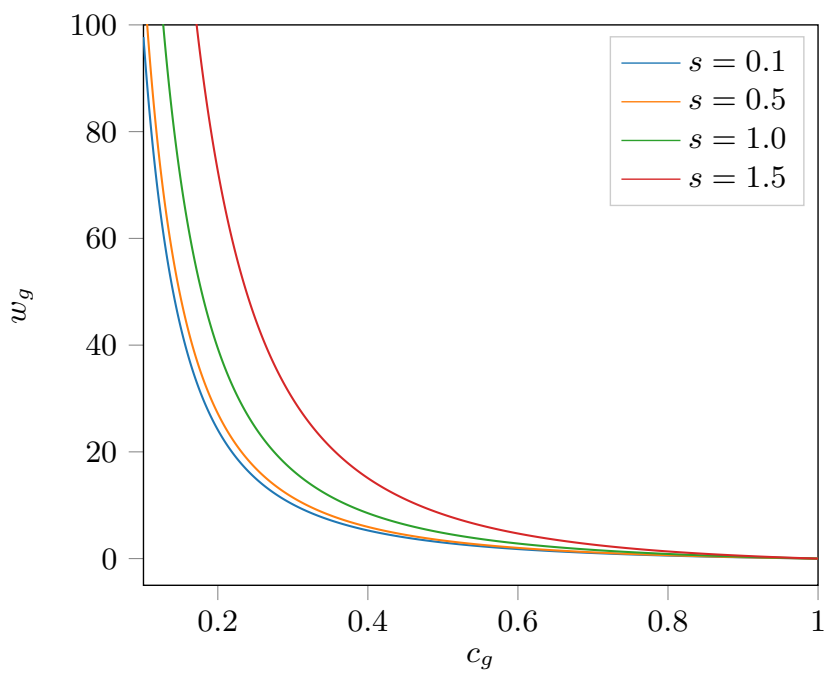

Fig. 1. Each line represents the weight $w_{g}$ as a function of $c_{g}$ for $N_{g}=1$ and four different values of the shape parameter $s$. This plot suggests that our weighting strategy gives high control over the low functional correlation case.

than in the case of high functional correlation by acting as a smooth threshold parameter, as shown in Figure 1.

Combining the COMMIT optimization problem defined in equation (1) together with the designed joint structural-functional prior offered by equations (3) and (2), we are now able to give the mathematical formulation of FIC.

Definition 1 (Functionally Informed COMMIT). Let $S$ be a set of $n$ fiber tracts and let $\mathcal{G}$ be the bundle structure over these fiber tracts. Moreover, let $A$ and $y$ be as in equation $1, \lambda>0, N_{g}$ equal to the cardinality of bundle $g$, $s>0$ and let $c_{g}>0$ be the functional correlation coefficient between the regions connected by bundle $g$. The solution of the following minimization problem is called FIC filtering of $S$.

$$
x^{*}=\underset{x \in \mathbb{R}_{+}^{n}}{\operatorname{argmin}} \frac{1}{2}\|A x-y\|_{2}^{2}+\lambda \sum_{g \in \mathcal{G}} \frac{1}{\sqrt{N_{g}}}\left(\frac{1}{c_{g}^{2}} \exp \left(\frac{1-c_{g}^{2}}{2 s^{2}}\right)-1\right)\left\|x_{g}\right\|_{2} .
$$

Theorem 1. The FIC filtering of a tractogram exists, is unique and can be obtained in polynomial time.

Proof. The target functional of equation (4) is strictly convex and lower semicontinuous, hence its solution exists and is unique. Moreover it can be split in a smooth part with $L$-Lipschitz continuous gradient and a non-smooth part for which we are able to compute the proximal operator [11], hence the solution can be approximated in polynomial time [2]. 


\section{Methods}

The two studied datasets were both generated with Phantomas [3] for the structural part (dMRI) and The Virtual Brain (TVB) [16] for the functional part (rs-fMRI). The diffusion MRI signal was simulated for a geometry of $94 \times 94 \times 94$ voxels of $(2.0 \mathrm{~mm})^{3}$ size in 64 sampling points uniformly distributed [4] on a single shell at $b=1500\left[\mathrm{~s} / \mathrm{mm}^{2}\right]$. The phantom consists of two streamline bundles either crossing or kissing in the center of the image. This is an extremely challenging configuration for tractography because voxels in the crossing/kissing region are identical for both configuration. See Figures 2 and 3 for a visual description of the simulated phantoms. On each of these datasets we ran a probabilistic anatomically constrained tractography algorithm seeding from the white matter. For each of the cortical regions we used TVB [16] to simulate the BOLD time series with repetition time (TR) of 1 second, obtaining a 15 minute time series for each region. The chosen noise model is Gaussian and additive with standard deviation equal to $5 \cdot 10^{-4}$, which corresponds to the one suggested in [18].

\subsection{Dynamic Functional Connectivity}

The wanted functional correlation coefficient corresponds to the dynamic functional connectivity ( $\mathrm{dFC}$ ) between two regions and is estimated using slidingwindow correlation. The window is defined by its length, its shape and the offset time. As far as the window length is concerned, we set it to 100 seconds following the strategy described in [12]. For the window shape we consider a rectangular box which gives unitary weight to each involved time point. The window offset is set to $1 \mathrm{TR}$ and the functional correlation between each pair of windows is estimated using the Pearson correlation coefficient. Finally, the maximal correlation among all the windows is elected to represent the wanted $\mathrm{dFC}$ coefficient.

\subsection{Parameters choice}

The experiments were run for the following values of parameters $\lambda$ and $s$ :

$$
\begin{gathered}
\lambda=[1.0,1.5,2.0,2.5], \\
s=[0.4,0.5,0.6,0.7,0.8,0.9,1.0,1.1,1.2,1.3,1.4,1.5] .
\end{gathered}
$$

\section{Results}

For each phantom we show the ground truth geometry and the solutions obtained with classical COMMIT, group sparsity (GS) and FIC in Figures 2 and 3. The ideal result preserves only the bundles that belong to the ground truth. It can be noticed how COMMIT and GS are not able to distinguish between true positive (TP) and false positive (FP) bundles, while FIC correctly detects the FPs and returns a properly filtered tractogram. This holds true for both the kissing bundles configuration and the crossing bundles configurations. For the 


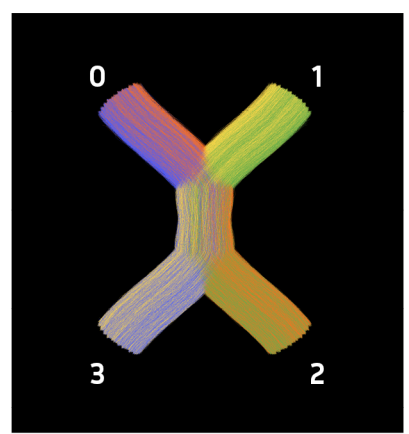

a) Geometry

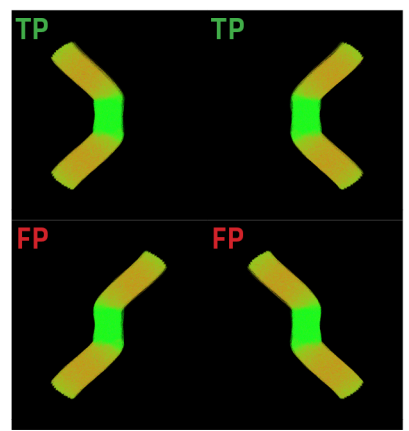

c) Group Sparsity

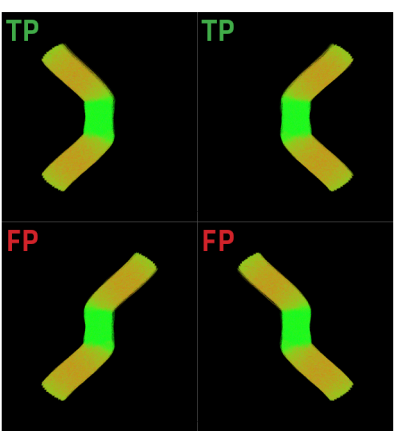

b) COMMIT

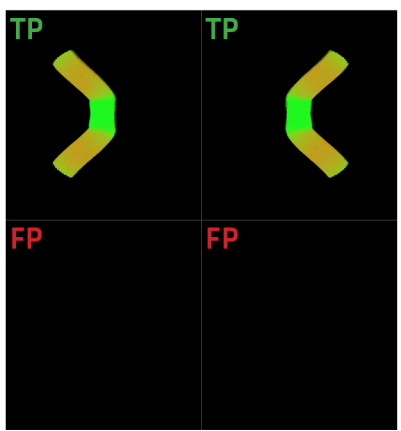

d) FIC

Fig. 2. Kissing. The shown results were computed for $\lambda=2.5$ and $s=1$. Subplot $a$ shows the ground truth geometry of the phantom, where each bundle has a distinctive color. Subplots $b, c$, and $d$ show every bundle belonging to the filtered tractogram. Those marked as FP should not be present after the tractography optimization while those marked as TP should be. Bundles connecting regions $0 \leftrightarrow 1$ and $2 \leftrightarrow 3$ were not generated by the tractography algorithm.

result given by each combination $\lambda$ and $s$ we computed two types of connectivity matrix. The first one is a classical streamline count and is indicated as $T$. The second one is what we call the FIC connectome, which is defined as $H_{i, j}=\left\|x_{g}^{*}\right\|_{2}$, where bundle $g$ connects regions $i$ and $j$ and $x^{*}$ is the solution obtained with FIC. Matrix $T$ is normalized by the total number of streamlines while $H$ is normalized by its maximal entry. Both of them share the same sparsity pattern, which reads as follows:

$$
G_{c}=\left[\begin{array}{cccc}
0 & 0 & * & 0 \\
0 & 0 & 0 & * \\
* & 0 & 0 & 0 \\
0 & * & 0 & 0
\end{array}\right] \quad G_{k}=\left[\begin{array}{cccc}
0 & 0 & 0 & * \\
0 & 0 & * & 0 \\
0 & * & 0 & 0 \\
* & 0 & 0 & 0
\end{array}\right]
$$

where $G_{c}$ is the sparsity pattern of the crossing configuration, while $G_{k}$ is the sparsity pattern of the kissing configuration. Due to different kind of normaliza- 


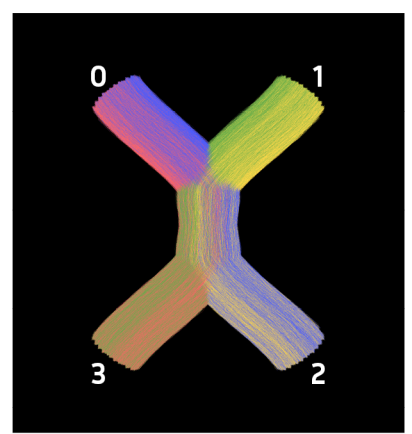

a) Geometry

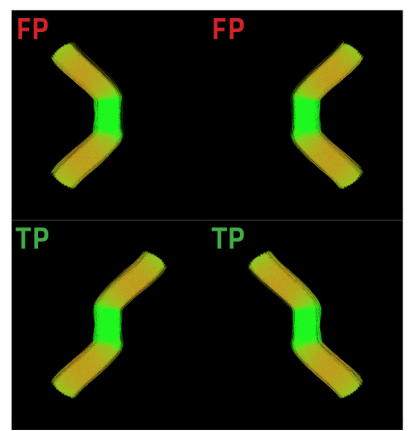

c) Group Sparsity

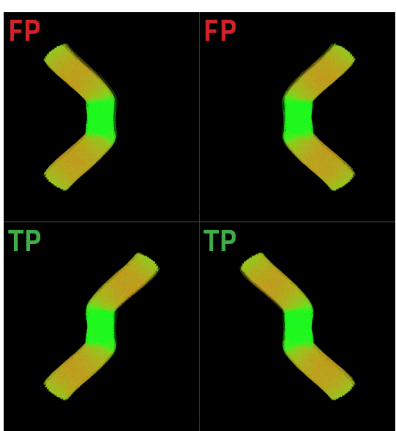

b) COMMIT

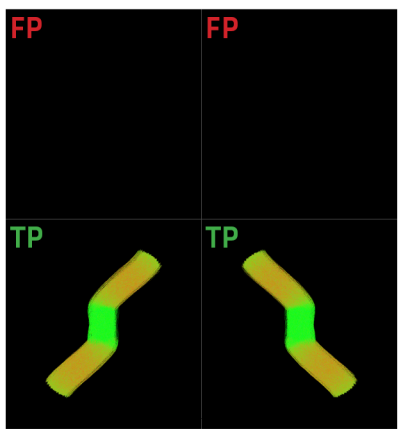

d) FIC

Fig. 3. Crossing. The shown results were computed for $\lambda=2.5$ and $s=1$. Subplot $a$ shows the ground truth geometry of the phantom, where each bundle has a distinctive color. Subplots $b, c$, and $d$ show every bundle belonging to the filtered tractogram. Those marked as FP should not be present after the tractography optimization while those marked as TP should be. Bundles connecting regions $0 \leftrightarrow 1$ and $2 \leftrightarrow 3$ were not generated by the tractography algorithm.

tion we expect the positive entries of $G(T)$ to be equal to 0.25 and the positive entries of $G(H)$ to be equal to 1.0. The mean and the standard deviation of the resulting connectivity matrices are reported in equation 8 for the FIC connectome and equation 9 for the streamline count of the crossing phantom. The analogue for the kissing phantom is shown in equation 10 and equation 11 respectively.

$$
\mu\left(H_{c}\right)=\left[\begin{array}{cccc}
0 . & 0 . & 1 . \pm 0 . & 0 . \\
0 . & 0 . & 0 . & 0.776 \pm 0.02 \\
1 . \pm 0 . & 0 . & 0 . & 0 . \\
0 . & 0.776 \pm 0.02 & 0 . & 0 .
\end{array}\right]
$$




$$
\begin{aligned}
& \mu\left(T_{c}\right)=\left[\begin{array}{cccc}
0 . & 0 . & 0.241 \pm 0.001 & 0 . \\
0 . & 0 . & 0 . & 0.259 \pm 0.001 \\
0.241 \pm 0.001 & 0 . & 0 . & 0 . \\
0 . & 0.259 \pm 0.001 & 0 . & 0 .
\end{array}\right] \\
& \mu\left(H_{k}\right)=\left[\begin{array}{cccc}
0 . & 0 . & 0 . & 0.99 \pm 0.007 \\
0 . & 0 . & 0.99 \pm 0.007 & 0 . \\
0 . & 0.99 \pm 0.007 & 0 . & 0 . \\
0.99 \pm 0.007 & 0 . & 0 . & 0 .
\end{array}\right] \\
& \mu\left(T_{k}\right)=\left[\begin{array}{cccc}
0 . & 0 . & 0 . & 0.255 \pm 0.001 \\
0 . & 0 . & 0.245 \pm 0.001 & 0 . \\
0 . & 0.245 \pm 0.001 & 0 . & 0 . \\
0.255 \pm 0.001 & 0 . & 0 . & 0 .
\end{array}\right]
\end{aligned}
$$

\section{Conclusions}

We proposed an evolution of the the recently proposed [5] bundle sparsity formulation of COMMIT that takes into account the functional information coming from rs-fMRI. The results obtained on two simple but very problematic phantoms prove that the FIC approach is effective for the detection of false positive connections in tractograms. Moreover these results are stable with respect to the two parameters to be tuned. We expect that the more complex configurations given by real data will need a deeper study of these parameters. Finally, we introduced a new concept of connectome that can't be considered neither purely structural nor purely functional but inherits features both from structural and functional information, hence it represents a first step towards the construction of a joint structural and functional connectome.

\section{Acknowledgement}

The authors would like to thank Rebecca Bonham-Carter for the help in simulating resting state networks. This work received funding from the European Research Council (ERC) under the European Union's Horizon 2020 research and innovation program (ERC Advanced Grant agreement No 694665 : CoBCoM Computational Brain Connectivity Mapping).

\section{References}

1. Bach, F., Jenatton, R., Mairal, J., Obozinski, G., et al.: Structured sparsity through convex optimization. Statistical Science 27(4), 450-468 (2012)

2. Beck, A., Teboulle, M.: A fast iterative shrinkage-thresholding algorithm for linear inverse problems. SIAM journal on imaging sciences 2(1), 183-202 (2009)

3. Caruyer, E., Daducci, A., Descoteaux, M., Houde, J.C., Thiran, J.P., Verma, R.: Phantomas: a flexible software library to simulate diffusion mr phantoms. In: Ismrm (2014) 
4. Caruyer, E., Lenglet, C., Sapiro, G., Deriche, R.: Design of multishell sampling schemes with uniform coverage in diffusion mri. Magnetic resonance in medicine 69(6), 1534-1540 (2013)

5. Daducci, A., Barakovic, M., Girard, G., Descoteaux, M., Thiran, J.P.: Reducing false positives in tractography with microstructural and anatomical priors. In: ISMRM 2018 - International Society for Magnetic Resonance in Medicine (2018)

6. Daducci, A., Dal Palú, A., Descoteaux, M., Thiran, J.P.: Microstructure informed tractography: pitfalls and open challenges. Frontiers in neuroscience 10, 247 (2016)

7. Daducci, A., Dal Palù, A., Lemkaddem, A., Thiran, J.P.: Commit: convex optimization modeling for microstructure informed tractography. IEEE transactions on medical imaging 34(1), 246-257 (2015)

8. Frigo, M., Barakovic, M., Thiran, J.P., Daducci, A.: Hierarchical tractography optimisation. In: CoBCoM 2017-Computational Brain Connectivity Mapping Winter School Workshop. p. 1 (2017)

9. Frigo, M., Gallardo, G., Costantini, I., Daducci, A., Wassermann, D., Deriche, R., Deslauriers-Gauthier, S.: Reducing false positive connection in tractograms using joint structure-function filtering. In: OHBM 2018-Organization for Human Brain Mapping (2018)

10. Girard, G., Fick, R., Descoteaux, M., Deriche, R., Wassermann, D.: Axtract: microstructure-driven tractography based on the ensemble average propagator. In: International Conference on Information Processing in Medical Imaging. pp. 675-686. Springer (2015)

11. Jenatton, R., Mairal, J., Obozinski, G., Bach, F.R.: Proximal methods for sparse hierarchical dictionary learning. In: ICML. pp. 487-494. No. 2010, Citeseer (2010)

12. Leonardi, N., Van De Ville, D.: On spurious and real fluctuations of dynamic functional connectivity during rest. Neuroimage 104, 430-436 (2015)

13. Maier-Hein, K.H., Neher, P.F., Houde, J.C., Côté, M.A., Garyfallidis, E., Zhong, J., Chamberland, M., Yeh, F.C., Lin, Y.C., Ji, Q., et al.: The challenge of mapping the human connectome based on diffusion tractography. Nature communications 8(1), 1349 (2017)

14. Panagiotaki, E., Schneider, T., Siow, B., Hall, M.G., Lythgoe, M.F., Alexander, D.C.: Compartment models of the diffusion $\mathrm{mr}$ signal in brain white matter: a taxonomy and comparison. Neuroimage 59(3), 2241-2254 (2012)

15. Pestilli, F., Yeatman, J.D., Rokem, A., Kay, K.N., Wandell, B.A.: Evaluation and statistical inference for human connectomes. Nature methods 11(10), 1058 (2014)

16. Sanz Leon, P., Knock, S.A., Woodman, M.M., Domide, L., Mersmann, J., McIntosh, A.R., Jirsa, V.: The virtual brain: a simulator of primate brain network dynamics. Frontiers in neuroinformatics 7, 10 (2013)

17. Smith, R.E., Tournier, J.D., Calamante, F., Connelly, A.: Sift2: Enabling dense quantitative assessment of brain white matter connectivity using streamlines tractography. Neuroimage 119, 338-351 (2015)

18. The Virtual Brain: The resting state network scripting tutorial (2018)

19. Zalesky, A., Fornito, A., Cocchi, L., Gollo, L.L., van den Heuvel, M.P., Breakspear, M.: Connectome sensitivity or specificity: which is more important? Neuroimage 142, 407-420 (2016) 